\title{
Comments on the algebraic properties of Dilaton actions $^{1}$
}

\section{A. Schwimmer ${ }^{a}$ and S. Theisen ${ }^{b}$}

\author{
${ }^{a}$ Weizmann Institute of Science, \\ Rehovot 76100, Israel \\ ${ }^{b}$ Max-Planck-Institut für Gravitationsphysik, Albert-Einstein-Institut, \\ 14476 Golm, Germany \\ E-mail: adam.schwimmer@weizmann.ac.il, stefan.theisen@aei.mpg.de
}

ABSTRACT: We study the relation between the dilaton action and sigma models for the Goldstone bosons of the spontaneous breaking of the conformal group. We argue that the relation requires that the sigma model is diffeomorphism invariant. The origin of the WZW terms for the dilaton is clarified and it is shown that in this approach the dilaton WZW term is necessarily accompanied by a Weyl invariant term proposed before from holographic considerations.

Keywords: AdS-CFT Correspondence, Conformal and W Symmetry, Anomalies in Field and String Theories

ARXIV EPRINT: 1311.4746

\footnotetext{
${ }^{1}$ Partially Supported by the Center for Basic Interactions of the Israeli Academy of Sciences. A.S. also acknowledges support from the Alexander von Humboldt-Foundation.
} 


\section{Contents}

1 Introduction 1

2 The Weyl invariant part of the dilaton sigma-model 3

$\begin{array}{lll}3 & \text { The WZW term } & 7\end{array}$

$\begin{array}{lll}4 & \text { Discussion } & 10\end{array}$

$\begin{array}{ll}\text { A Review of trace anomalies in holography } & 11\end{array}$

\section{Introduction}

Chiral anomalies are well understood algebraically [1, 2]. Their general form can be obtained by considering the theory in $d=2 n$ as a boundary of a $d+1$ dimensional manifold. The action in $d+1$ dimensions is the local Chern-Simons action and since this action is gauge invariant only up to a boundary term the correct anomaly is reproduced by this boundary term. In a sense this could be considered as a manifestation of "holography".

When the chiral symmetry is spontaneously broken Goldstone bosons are present. Since the Goldstone bosons have to reproduce the chiral anomalies, specific features of their action ("sigma-model" in the following) follow from the aforementioned structure: besides a local term in $d=2 n$ dimensions which realizes nonlinearly the symmetry there is a second term [3, 4] ("WZW term" in the following) which lives in $d+1$ dimensions and reproduces the anomaly through the above mechanism.

In the present note we want to study in detail the analogous problems for trace anomalies. Following the explicit calculation of the trace anomalies in the AdS/CFT duality [5] it was realized [6] that a mechanism rather analogous to the one described above for chiral anomalies is at work: the gravitational action in $d+1$ dimension plays the role of the Chern-Simons action and a particular subgroup of the $d+1$-dimensional diffeomorphism acts as the analogue of the gauge transformations producing the anomalies at the boundary.

When the conformal symmetry is spontaneously broken the Goldstone boson (in the following "the dilaton") should reproduce the trace anomalies [7-9]. The effective action with this property can be constructed and a WZW term appears. Compared with the general properties of the chiral Goldstone bosons action outlined above the dilaton action has strange features: the WZW term is local directly in $d=2 n$ and does not seem to have any higher dimensional origin.

In order to understand this feature we rely on the basic distinguishing property of the dilaton: even though the spontaneous breaking of the conformal symmetry in Euclidean signature is the breaking of the $\mathrm{SO}(\mathrm{d}+1,1)$ group to $\mathrm{SO}(\mathrm{d}) \times \mathrm{T}_{\mathrm{d}}$ there is only one Goldstone 
boson, the dilaton [10-12]. This is of course a consequence of the fact that all the conformal currents can be constructed in terms of the energy momentum tensor, their conservation being the consequence of tracelessness. The gauging of the $\mathrm{SO}(\mathrm{d}+1,1)$ currents is replaced by diffeomorphism invariance and by the Weyl symmetry.

In the broken phase one would start naively with a sigma-model on the $\mathrm{SO}(\mathrm{d}+$ $1,1) /\left[\mathrm{SO}(\mathrm{d}) \times \mathrm{T}_{\mathrm{d}}\right]$ coset. We propose that the reduction from the $d+1$ fields parametrizing the coset to the single dilaton is achieved by a special new, characteristic feature of the sigma-model: diffeomorphism invariance in $d=2 n$ dimensions for the invariant term and in $d+1$ dimensions for the WZW term, respectively. By choosing a particular parametrization the coset coordinates are reduced to a single field and the WZW term becomes explicitly local.

We will formulate the sigma-model in a general metric background. Since the input $d$-dimensional metric should give rise in the sigma-model to a metric depending on $d+1$ coordinates - the Goldstone boson fields - we are led from the beginning to consider a "holographic" setup. Moreover, the metric in the sigma-model action should admit the action of a group isomorphic to the Weyl group which makes the connection to holography even stronger. In spite of that the freedom for dilaton actions constrained by the algebraic approach is much larger than the one which follows from a strict application of holography. In particular, as we will discuss in detail, there is no relation between the $d+1$ dimensional actions and solutions we are using in the construction.

Applying the above mentioned procedure both for the invariant terms of the dilaton action and the one reproducing the trace anomalies (the "WZW" part) we get an interesting connection between the two once one imposes the condition that there is no potential for the dilaton, a necessary condition for the spontaneous breaking of conformal invariance. Our conclusion is that the special action proposed for the dilaton in a holographic setup in [13] has a general algebraic origin being normalized by the " $a$ " trace anomaly whenever conformal invariance is spontaneously broken.

The paper is organized as follows:

In section 2 we construct the $d$ dimensional part of the reparametrization invariant sigma-model and we show how it reduces to the Weyl invariant part of the dilaton action. In section 3 we construct the $d+1$ dimensional reparametrization invariant WZW term and we reduce it to the dilaton WZW term. We discuss the relation between invariant terms and the WZW terms following from the requirement of vanishing potential for the dilaton. The relations between WZW terms corresponding to different even dimensions is made explicit. In the last section we discuss various applications of the formalism developed and possible generalizations. In appendix A we review the holographic calculations of trace anomalies and the realization of Weyl symmetry in holography which motivate the choices of the explicit metric backgrounds in sections 2 and 3.

Related and complementary discussions of some of the aspects addressed here can be found in [14-18]. While these references rely on supersymmetry, this is not assumed here. 


\section{The Weyl invariant part of the dilaton sigma-model}

We will follow here an algebraic approach though, as we will see, the results have an immediate holographic interpretation.

Consider in $d$ dimensions the breaking of the conformal group $\mathrm{SO}(\mathrm{d}+1,1)$ to the Poincaré group $\mathrm{SO}(\mathrm{d}) \times \mathrm{T}_{\mathrm{d}}$. The coset of Goldstone bosons can be parametrized by $d+1$ fields $X^{\mu}\left(x^{i}\right)$ where $\mu=1, \ldots, d+1$ and $i=1, \ldots, d$. The metric on the space of the $X$ fields is $A d S_{d+1}$ with isometry $\mathrm{SO}(\mathrm{d}+1,1)$ such that the broken isometries are nonlinearly realized.

Since we want to construct the analogue of the "gauged sigma-model" we allow a more general metric $G_{\mu \nu}\left(X^{\tau}\right)$ on which the Weyl transformations act. The condition this metric has to fulfill in order to serve our purposes are:

a) It should be a $d+1$ metric but a functional of a $d$ dimensional metric $g_{i j}, i, j=1, \ldots, d$

$$
G_{\mu \nu}=F_{\mu \nu}\left[g_{i j}\right]
$$

b) It should admit the action of a group isomorphic to the Weyl transformations such that

$$
\delta_{\sigma} G=F\left[g_{i j} \exp 2 \sigma(x)\right]-F\left[g_{i j}\right]
$$

where $\delta_{\sigma}$ denotes the action on the $d+1$ dimensional metric isomorphic to the Weyl transformations.

c) For $g_{i j}=\delta_{i j}$ it should reduce to the natural metric on the $\frac{\mathrm{SO}(\mathrm{d}+1,1)}{\mathrm{SO}(\mathrm{d}) \times \mathrm{T}_{\mathrm{d}}}$ coset which is $A d S_{d+1}$.

A class of metrics which satisfy the above requirements are solutions of $d+1$ dimensional "bulk" actions which admit $A d S_{d+1}$ solutions, specified by the boundary metric $g_{i j}$ (which in the continuation we will denote by $g_{i j}^{(0)}$ ) in the Fefferman-Graham gauge. Obviously this class satisfies the above requirements, the group action isomorphic to Weyl transformations being the PBH transformations as explained in the appendix.

The connection to holography is now obvious though we stress that we will use only the algebraic properties of the solution. In particular the specific action to which the metric is a solution will not play a role. It is an interesting question if there are metrics which satisfy the above requirements not arriving from a holographic construction.

The natural building blocks for the gauged sigma-model are the induced metric:

$$
h_{i j}(x)=G_{\mu \nu}\left(X^{\tau}(x)\right) \partial_{i} X^{\mu}(x) \partial_{j} X^{\nu}(x)
$$

and the second fundamental form. While for an ordinary sigma-model we would take as an action e.g. $\delta^{i j} h_{i j}$, here we insist on reparametrization invariance in $d$ dimensions which, together with the field redefinition invariance present in (2.3), will allow us to project to the dilaton. Therefore the minimal sigma-model action having these properties is:

$$
S=\frac{1}{\ell^{d}} \int d^{d} x \sqrt{\operatorname{det} h_{i j}} \text {. }
$$


If from the beginning we choose $G_{\mu \nu}$ to be in the FG gauge, we split the fields $X^{\mu}$ into $X^{i}(x) i=1, \ldots, d$ and $\Phi(x)$ in the " $\rho$ "-direction. Now we can achieve the reduction to the dilaton action by choosing the gauge:

$$
X^{i}(x)=x^{i}
$$

and the gauge fixed action becomes:

$$
S=\frac{1}{\ell^{d}} \int d^{d} x \frac{\sqrt{\operatorname{det} g_{i j}(x, \Phi(x))}}{\Phi^{d / 2}(x)} \sqrt{1+\frac{\ell^{2} g^{i j}(x, \Phi(x)) \partial_{i} \Phi \partial_{j} \Phi(x)}{4 \Phi(x)}} .
$$

For the particular case of the AdS metric we recognize the expression proposed in [13] as representing the action for the displacement of the brane which breaks the gauge and conformal symmetry on the $N=4$ Super Yang-Mills Coulomb branch.

In order to exploit the symmetries of the action it is convenient to start with its unfixed form (2.4). Since in the FG gauge the metric is determined by its boundary value $g_{i j}^{(0)}(X)$, the action is a functional of $g_{i j}^{(0)}$ and $X^{\mu}$. The action has symmetries of two kinds:

a) Field transformations of the $X^{\mu}$ fields relating two different background metrics $G_{\mu \nu}$. These transformation make explicit the variation under a change of $g_{i j}^{(0)}$. The transformations are inherited from residual gauge transformations in the FG gauge, i.e. the PBH transformation parametrized by $\sigma\left(X^{j}\right)$ and $X^{j}$-dependent field transformations parametrized by $\zeta^{i}\left(X^{j}\right)$. We rewrite the PBH transformations of the appendix, making it explicit that in the framework of the sigma-model we deal with field transformations at fixed coordinates $x^{i}$ :

$$
\begin{aligned}
\Phi^{\prime} & =\Phi\left(1+2 \sigma\left(X^{j}\right)\right) \\
X^{\prime i} & =X^{i}-a^{i}\left(X^{j}, \Phi(x)\right)-\zeta^{i}\left(X^{j}\right)
\end{aligned}
$$

where

$$
a^{i}(X, \Phi(x))=\frac{\ell^{2}}{2} \int_{0}^{\Phi(x)} d \rho^{\prime} g^{i j}\left(x, \rho^{\prime}\right) \partial_{j} \sigma(X) .
$$

Such a transformation changes $g_{i j}^{(0)}$ by:

$$
\delta g_{i j}^{(0)}\left(X^{k}\right)=2 \sigma\left(X^{k}\right) g_{i j}^{(0)}\left(X^{k}\right)+\nabla_{i} \zeta_{j}\left(X^{k}\right)+\nabla_{j} \zeta_{i}\left(X^{k}\right)
$$

where the covariant derivatives are constructed with $g_{i j}^{(0)}$ and all the functional dependences are on $X^{j}$.

b) Reparametrizations of the $x^{i}$ variables parametrized by $\xi^{i}\left(x^{k}\right)$ :

$$
x^{i}=x^{i}-\xi^{i}\left(x^{k}\right)
$$

under which the "fields" $X^{\mu}$ transform as:

$$
\delta X^{\mu}\left(x^{k}\right)=\xi^{i} \partial_{i} X^{\mu}\left(x^{k}\right) .
$$

We are now ready to study the symmetries of the "projected" action (2.6) in the special coordinates $(2.5)$, the action being now a functional just of $g_{i j}^{(0)}$ and $\Phi$. 
After the transformation (2.7) the special choice (2.5) is not anymore respected. In order to reinstate $i^{1}{ }^{1}$ we should make a reparametrization with the special choice of the parameters $\xi^{i}$ :

$$
\xi^{i}\left(x^{k}\right)=a^{i}\left(X^{k}=x^{k}, \Phi(x)\right)+\zeta^{i}\left(X^{k}=x^{k}\right)
$$

Therefore the action (2.6) will be invariant under a joint transformation:

$$
\delta g_{i j}^{(0)}(x)=2 \sigma(x) g_{i j}^{(0)}+\nabla_{i} \zeta_{j}+\nabla_{j} \zeta_{i}
$$

and

$$
\delta \Phi(x)=2 \sigma(x) \Phi(x)+\left[a^{i}\left(X^{k}=x^{k}, \Phi(x)\right)+\zeta^{i}\left(X^{k}=x^{k}\right)\right] \partial_{i} \Phi(x)
$$

where we indicated the places where the field $X^{k}$ was replaced with the variables $x^{k}$ using the gauge (2.5).

Equations (2.14), (2.15) make explicit the invariance of (2.6) under the Weyl transformations. The transformations are non-anomalous since there is no boundary term which could be the source of violation in the above classical argument.

Equation (2.15) shows that in order to have the usual transformation of the dilaton field $\tau$ under Weyl transformation:

$$
\tau \rightarrow \tau+\sigma
$$

there is a field redefinition relating $\Phi$ and $\tau$. Using (2.15) the field redefinition can be found iteratively giving an expansion in powers of $e^{2 \tau}$ where the terms of $\mathcal{O}\left(e^{2 n \tau}\right)$ contain $2(n-1)$ derivatives. $^{2}$ The 0 -th order solution of $(2.15)$ is:

$$
\Phi^{(0)}=e^{2 \tau} .
$$

For the higher order terms in the iterative solution we make the most general Ansatz and require (2.15) to be satisfied. (For simplicity and without loss of generality, we set $\zeta^{i}=0$.) To proceed we need the $\Phi$-expansion of $a^{i}$ which depends on the higher order terms in the FG expansion of the metric. Both can be found in [20]. In this way we find

$$
\frac{1}{\ell^{2}} \Phi^{(1)}=\frac{1}{2} e^{4 \tau}(\nabla \tau)^{2}+\alpha e^{2 \tau} \hat{R}
$$

and

$$
\begin{aligned}
& \frac{1}{\ell^{4}} \Phi^{(2)}=e^{6 \tau}\left(\frac{R^{i j} \nabla_{i} \tau \nabla_{j} \tau}{4(d-2)}-\frac{R(\nabla \tau)^{2}}{8(d-1)(d-2)}+\frac{1}{4} \nabla^{i} \tau \nabla^{j} \tau \nabla_{i} \nabla_{j} \tau+\frac{7}{16}(\nabla \tau)^{4}\right) \\
& +\alpha e^{2 \tau}\left(\frac{1}{2} \hat{\nabla}^{i} \tau \hat{\nabla}_{i} \hat{R}+\hat{\nabla}^{i} \tau \hat{\nabla}_{i} \tau \hat{R}\right)+e^{2 \tau}\left(\beta_{1} \hat{\square} \hat{R}+\beta_{2} \hat{R}^{2}+\beta_{3} \hat{R}^{i j} \hat{R}_{i j}+\beta_{4} \hat{C}^{i j k l} \hat{C}_{i j k l}\right)
\end{aligned}
$$

Here $\alpha$ parametrizes the homogeneous solution of $(2.15)$ at $\mathcal{O}\left(\partial^{2}\right)$ and $\beta_{1}, \ldots, \beta_{4}$ parameterizes the homogeneous solutions at $\mathcal{O}\left(\partial^{4}\right)$. The hatted quantities are built from the invariant metric $\hat{g}_{i j}=e^{-2 \tau} g_{i j}$ and $g_{i j}=g_{i j}^{(0)}$.

\footnotetext{
${ }^{1}$ For a related discussion see [19].

${ }^{2}$ The discussion here generalizes that of [19] to a curved metric.
} 
Plugging this into (2.6) we obtain the "universal minimal" invariant part of the action (cf. appendix A):

$$
S=\frac{1}{\ell^{d}} \int d^{d} x \sqrt{\hat{g}}\left(1+\frac{\ell^{2}}{4(d-1)} \hat{R}+\frac{\ell^{4}}{32(d-2)(d-3)}\left(\hat{E}_{4}-\hat{C}^{2}\right)+\mathcal{O}\left(\partial^{6}\right)\right)
$$

Additional terms can be obtained by adding to (2.4) reparametrization invariant terms depending on curvatures constructed from $h_{i j}$ as well as from the second fundamental form. ${ }^{3}$ Also, we have set $\alpha=\beta_{i}=0$ (cf. (2.18), (2.19)). Note that in $d=2$ the $\mathcal{O}\left(\partial^{2}\right.$ ) terms are $\frac{1}{4} \int \sqrt{g} R$ while in $d=4$ the $\mathcal{O}\left(\partial^{4}\right)$ terms are $\frac{1}{64} \int \sqrt{g}\left(E_{4}-C^{2}\right)$.

If one keeps the homogeneous terms in $\Phi$, one finds the additional terms

$$
\begin{aligned}
\Delta S=\frac{1}{\ell^{d}} \int d^{d} x \sqrt{\hat{g}}\{ & -\frac{d \ell^{2}}{2} \alpha \hat{R}+\frac{\ell^{4}}{8} d(d+2) \alpha \hat{R}^{2}+\frac{\ell^{4}}{8} \frac{(d-2)}{(d-1)} \alpha^{2} \hat{R}^{2} \\
& \left.-\frac{d \ell^{4}}{2}\left(\beta_{1} \hat{\square} \hat{R}+\beta_{2} \hat{R}^{2}+\beta_{3} \hat{R}^{i j} \hat{R}_{i j}+\beta_{4} \hat{C}_{i j k l} \hat{C}^{i j k l}\right)\right\} .
\end{aligned}
$$

The above analysis made for the minimal action can be repeated after adding terms depending on curvatures built with the induced metric and/or the second fundamental form. From the analysis above it is clear that while the final form of the action will depend on these terms the field redefinition of $\Phi$ in terms of $\tau$ is universal. A unique feature of the minimal action which we will use in the following is that it is the only term written in terms of the $\Phi$ field which contains a "potential" of the dilaton field $\tau$. In flat space this is simply $\frac{1}{\Phi^{d / 2}}$, while after the field redefinition it gives rises to the $\sqrt{\hat{g}}$ term.

Another application of our formalism is the study of the symmetries of (2.6) for the special case $g_{i j}^{(0)}=\delta_{i j}$, i.e. AdS background metric. The AdS metric is invariant under special conformal transformations accompanied by an appropriate Weyl transformation, i.e. in the notation of (2.7):

$$
\zeta^{i}=\frac{1}{2} \epsilon^{i} x^{2}-x^{i}(\epsilon \cdot x)
$$

with $\epsilon^{i}=$ const. and

$$
\sigma(x)=-\frac{1}{d}(\partial \cdot \zeta)=\epsilon \cdot x
$$

For a flat background the $a^{i}$ have a very simple form:

$$
a_{i}=\frac{\ell^{2}}{2} \Phi(x) \partial_{i} \sigma(x)=\frac{\ell^{2}}{2} \epsilon_{i} \Phi(x) .
$$

Therefore (2.6) for a flat $g_{i j}^{(0)}$ is invariant under a reparametrization transformation:

$$
x^{i}=x^{i}-\frac{1}{2} \epsilon^{i} x^{2}+x^{i}(\epsilon \cdot x)-\frac{\ell^{2}}{2} \epsilon^{i} \Phi(x)
$$

followed by a field transformation:

$$
\Phi^{\prime}\left(x^{\prime}\right)=\Phi(x)+2 \epsilon \cdot x \Phi(x)
$$

which is the defining symmetry of (2.6) in [13].

\footnotetext{
${ }^{3}$ Such terms were considered, in a somewhat different context, in [21].
} 


\section{The WZW term}

We want to construct a sigma-model term which reproduces the trace anomalies. This should be an action on a $d+1$ dimensional manifold with boundary and should share with the invariant term the property of reducing to a functional just of the dilaton field and the boundary metric. We will achieve that by requiring that the action is invariant under reparametrizations in $d+1$ dimensions. We define

$$
f_{\alpha \beta}=G_{\mu \nu}(X) \partial_{\alpha} X^{\mu} \partial_{\beta} X^{\nu}
$$

where $G_{\mu \nu}$ is the bulk metric in FG gauge and $\alpha, \beta=1, \ldots, d+1$. The fields $X^{\mu}$ depend now on $d+1$ coordinates which we split from the beginning into $x^{i}, i=1, \ldots, d$ and $\rho$. The boundary of the manifold is at $\rho=0$. The WZW action is then

$$
S_{\mathrm{WZW}}=\frac{1}{\ell^{d}} \int d^{d} x d \rho \sqrt{\operatorname{det} f_{\alpha \beta}} .
$$

We remark that the classical solution metric which is used in (3.1) should not be necessarily a solution of a minimal action like (3.2): only its transformation properties enter our arguments.

Since the embedding has the same dimension as the space all the relevant information is in the boundary conditions of the embedding fields. We split $X^{\mu}$ into $X^{i}(x, \rho)$ and $\Phi(x, \rho)$. The symmetries of the action are again:

a) Field transformations relating backgrounds defined by different $g_{i j}^{(0)}$. These transformations, which involve fields at fixed coordinates, will have exactly the same form as in the previous section, i.e. (2.7) and (2.9).

b) Reparametrizations in $d+1$ dimensions.

We choose again a special set of coordinates by:

$$
X^{i}(x, \rho)=x^{i} .
$$

Then the action (3.2) becomes:

$$
S_{\mathrm{WZW}}=\frac{1}{2 \ell^{d}} \int d^{d} x d \rho \partial_{\rho} \Phi \frac{\sqrt{\operatorname{det} g_{i j}(x, \Phi(x, \rho))}}{\Phi(x, \rho)^{1+d / 2}} .
$$

We see that a change of variable between $\rho$ and $\Phi(x, \rho)$ is possible in the integral at each fixed $x$ such that the action (3.4) depends on $\Phi(x, \rho)$ just through its boundary value $\Phi(x, \rho=0)$.

The symmetry transformations which leave the gauge condition (3.3) unchanged are again PBH transformations accompanied by reparametrizations:

$$
\delta g_{i j}^{(0)}(x)=2 \sigma(x) g_{i j}^{(0)}+\nabla_{i} \zeta_{j}(x)+\nabla_{j} \zeta_{i}(x)
$$

and

$$
\delta \Phi(x, \rho)=2 \sigma(x) \Phi(x, \rho)+\left[a^{i}\left(X^{k}=x^{k}, \Phi(x, \rho)\right)+\zeta^{i}\left(X^{k}=x^{k}\right)\right] \partial_{i} \Phi(x, \rho)
$$


$\sigma(x)$ and $\zeta^{i}(x)$ being the parameters of the transformation. We remark that as in the previous section $a^{i}$ are determined by the $\mathrm{PBH}$ transformations such that $\Phi$ is treated just as an expansion parameter, its $x$-dependence not being acted upon.

At the boundary $\rho=0, \Phi(x, \rho=0)$ has exactly the same Weyl transformation property as $\Phi(x)$ in the previous section and the field redefinition relating it to the dilaton field $\tau(x)$ is identical.

As long as $\Phi(x, 0)$ and $g_{i j}^{(0)}$ are transformed simultaneously the action will be invariant. This is the analogue in this set up of the fact that whatever the generating functional of a CFT $W(g)$ is, if we define $\hat{g}_{i j}=e^{-2 \tau} g_{i j}$ then $W(\hat{g})$ will be invariant under a joint transformation of the metric and the dilaton. The expression which is local and reproduces the anomalies i.e. the WZW term, is the difference $W(g)-W(\hat{g})$.

From (3.6) it is clear that in flat space $\tau=0$ corresponds to $\Phi(x, \rho=0)=1$. Therefore, using the change of variable from $\rho$ to $\Phi(x, \rho)$ we define the WZW action by

$$
S_{\mathrm{WZW}}=\frac{1}{2 \ell^{d}} \int_{1}^{\Phi(x, 0)} d \Phi d^{d} x \frac{\sqrt{\operatorname{det} g_{i j}(x, \Phi)}}{\Phi^{1+d / 2}} .
$$

We expect that the expression (3.7) has a well defined limit for $d \rightarrow 2 n$.

We discuss now the way (3.7) reproduces the trace anomalies. Under (3.5), (3.6) the metric $g_{i j}^{(0)}$ transforms by a Weyl transformation. If this transformation is accompanied by the appropriate transformation of $\Phi$ or equivalently of $\tau$, this will be an invariance. Therefore the variation will not get a contribution from the upper limit of integration. On the other hand for the lower limit of integration the expression would be invariant if the lower limit which corresponds to $\Phi(x, 0)=1$ transformed as follows from (3.6), i.e. by the amount

$$
\delta(\Phi=1)=2 \sigma(x) .
$$

Since the lower limit is kept fixed the variation is the compensating contribution coming from the integrand multiplied with (3.8):

$$
\delta S_{\mathrm{WZW}}=-\frac{1}{2 \ell^{d}} \int d^{d} x 2 \sigma(x) \sqrt{\operatorname{det} g_{i j}(x, \Phi=1)} .
$$

As discussed in the appendix, in the expression (3.9) the anomalies in $d=2 n$ are to be found among the terms with no $\ell$ dependence. With this identification it is clear that the type A anomalies [22] following from (3.9) are the ones calculated a long time ago [5, 20].

One can now explicitly work out the WZW part in the dilaton action in an external metric following from (3.7). Using the FG expansions for $g_{i j}(x, \rho)$ in [5] and [20] we find in $d=4$ :

$$
\begin{aligned}
S_{\mathrm{WZW}} & =\int \sqrt{g} d^{4} x\left(\frac{1}{4 \ell^{4}}\left(1-e^{-4 \tau}\right)-\frac{1}{24 \ell^{2}} R\left(1-e^{-2 \tau}\right)+\frac{1}{4 \ell^{2}}(\partial \tau)^{2} e^{-2 \tau}\right. \\
& +\frac{1}{64}\left(\tau E_{4}+4\left(R^{i j}-\frac{1}{2} R g^{i j}\right) \nabla_{i} \tau \nabla_{j} \tau-4(\nabla \tau)^{2} \square \tau+2(\nabla \tau)^{4}\right)+\left(\frac{\gamma}{2}-\frac{1}{64}\right) \tau C^{2} .
\end{aligned}
$$


Here $\gamma$ is a combination of the two parameters which specify the homogeneous solution of the PBH transformation for $g^{(2)}[20]$. They could be fixed by explicitly solving the equations of motion of the bulk gravitational action. For the Einstein-Hilbert action with cosmological constant, which is dual do $\mathcal{N}=4$ SYM theory, $\gamma=0$ and one finds that the two anomaly coefficients $a$ and $c$ are the same. In deriving (3.10) we have set to zero the free parameters which appear in the solution of (3.6), i.e the coefficients $\alpha$ and $\beta_{i}$ in (2.18) and (2.19).

An alternative way of writing (3.10) is

$$
\begin{aligned}
& S_{\mathrm{WZW}}=\int d^{4} x\left\{-\frac{1}{4 \ell^{2}}(\sqrt{\hat{g}}-\sqrt{g})+\frac{1}{24 \ell^{2}}(\sqrt{\hat{g}} \hat{R}-\sqrt{g} R)\right. \\
& \left.\quad+\frac{1}{64}\left(\tau E_{4}+4\left(R^{i j}-\frac{1}{2} R g^{i j}\right) \nabla_{i} \tau \nabla_{j} \tau-4(\nabla \tau)^{2} \square \tau+2(\nabla \tau)^{4}\right)+\left(\frac{\gamma}{2}-\frac{1}{64}\right) \tau C^{2}\right\} .
\end{aligned}
$$

The expression (3.11) above shows clearly that besides the standard terms of the dilaton action which reproduce the trace anomaly there are invariant terms including the "dilaton potential" term $\sqrt{\hat{g}}$ whose normalization is completely fixed by the "a" anomaly. In appendix A this fact is analysed in detail showing that it has an algebraic origin and it is independent both on the metric used and a possible replacement of the "minimal action" (3.7) by an action having additional curvature terms.

As shown in the previous section the action (2.6) is the only one among the invariant actions which has a potential term for the dilaton. Since the total effective action for the dilaton cannot have a potential term for the dilaton this term has to be cancelled by the one appearing in the WZW part (3.11). We conclude therefore that (2.6) with a normalization " $-a$ " should necessarily accompany (3.11). This unexpected conclusion is a consequence of our embedding the dilaton in the space of the $d+1$ Goldstone bosons with the symmetry structure assumed. In the normalization of the type A anomaly coefficient s.t. $\delta_{\sigma} W=a \int \sigma E_{4}+$ type $\mathrm{B}$, the total dilaton action is

$$
16 a\left(S+4 S_{\mathrm{WZW}}\right) \text {. }
$$

In this combination the dilaton potential cancels and the anomaly is reproduced with the correct normalization. The same relative factor was derived in [13] from the requirement of supersymmetry in the form of a no-force requirement for a probe D3-brane in $A d S_{5}$.

Alternative arguments for the special role of (2.6) were recently put forward in [18].

If we want to calculate the WZW terms $S_{\mathrm{WZW}}^{(0)}$, i.e. the dilaton self-interaction terms in flat external metric from (3.7) with $g_{i j}^{(0)}=\delta_{i j}$, we obtain a deceptively simple looking universal expression:

$$
S_{\mathrm{WZW}}^{(0)}=-\frac{1}{d \ell^{d}} \int d^{d} x\left(\frac{1}{\Phi(x, 0)^{d / 2}}-1\right) .
$$

For each dimension we should look at the term independent on $\ell$. In principle this calculation should check the relative normalizations of the terms in different dimensions following from the general relation between type A anomalies [6, 20]. 


\section{Discussion}

The special feature we used for constructing the sigma-model actions which reduced to the dilaton action was their diffeomorphism invariance. Using this invariance we could gauge away some of the "would be Goldstone bosons" ending with the relevant one, the dilaton. We believe that this is a general feature whenever a space-time symmetry is spontaneously broken. It would be interesting to have a general treatment of this pattern. ${ }^{4}$

For the dilaton action the embedding gave new, unexpected information, i.e. that there is a Weyl invariant piece of the action, the "minimal" one normalized by the $a$ anomaly which should accompany the WZW term. This feature appeared when we formulated the actions in term of the $\Phi$ field, the coordinate left unfixed after using diffeomorphism invariance. The change of variable to the $\tau$ field, which realizes additively the Weyl transformation, obscures this connection. It is an open question if one can reformulate in an invariant fashion the "minimal" action in terms of the $\tau$ field.

We used extensively the "gauging" of the sigma-model in order to study its symmetries. The gauging used was a coupling to a general $d$ dimensional metric. We used the fact that the natural $A d S_{d+1}$ metric on the Goldstone boson space can be related in the Poincaré patch to a $d$ dimensional flat metric. Our "gauging" was a natural deformation of this relation leading to a natural appearance of the holographic set up. Alternative paths for coupling the sigma-model to a metric, still respecting diffeomorphism invariance should be explored. Once the above structure was picked we were led to consider metrics which were solutions of equations of motion corresponding to a bulk action. There is a large freedom in the choice of the bulk action and in particular there is no need that the action picked for the sigma-model has any relation to the action which provided the metric solution. In this sense the treatment seems to be purely algebraic. Nevertheless one should investigate if this very relaxed holographic framework is really necessary or the requirements we listed for the metrics can be achieved in a different way.

At a more basic level the gauging we used via the coupling to a metric might not be the most natural one. Following the analogy to the chiral situation a coupling of the sigmamodel to $\mathrm{SO}(\mathrm{d}+1,1)$ gauge fields would be more natural. This would require however the understanding of the relation (if any) between the $a$ Weyl anomaly and descent equations of the conformal group.

\section{Acknowledgments}

Useful discussions with O. Aharony and S. Yankielowicz are gratefully acknowledged. We in particular thank Z. Komargodski for his collaboration at the early stages of this project and for his helpful comments throughout. S.T. thanks S. Kuzenko for the invitation, hospitality and discussions at Western Australian University in Perth during the final stages.

\footnotetext{
${ }^{4}$ This is similar in spirit to e.g. [11, 12], but in these references backgrounds with isometries are considered while here no such assumption is made.
} 


\section{A Review of trace anomalies in holography}

We will adopt the point of view that while AdS/CFT duality gives a physical realization in which the trace anomalies of a CFT appear, one can abstract from it general, algebraic properties rather similar to the "descent treatment" [1] of chiral anomalies.

The basic setup involves a $d+1$ (where $d=2 n$ ) dimensional manifold with the topology of the Poincaré patch of AdS. For the $d$ dimensional boundary we will take a manifold with Euclidean signature and the topology of $\mathbb{R}^{d}$ (taking the topology to be e.g. $S^{d}$ is unimportant as long as we discuss local anomalies).

The metric $G_{\mu \nu}$ on the $d+1$ dimensional manifold can be brought to the "FeffermanGraham (FG in the following) gauge" ideally suited for the problem:

$$
d s^{2}=G_{\mu \nu} d X^{\mu} d X^{\nu}=\frac{\ell^{2}}{4}\left(\frac{d \rho}{\rho}\right)^{2}+\frac{1}{\rho} g_{i j}(x, \rho) d x^{i} d x^{j} .
$$

Here $\mu, \nu=1, \ldots, d+1$ and $i, j=1, \ldots, d$. The coordinates are chosen such that $\rho=0$ corresponds to the boundary. We will assume that $g_{i j}$ is regular at $\rho=0$.

We will consider $G_{\mu \nu}$, which are solutions of a gravitational equation of motion determined through the FG expansion in terms of the boundary value $g_{i j}^{(0)}(x)=g_{i j}(x, \rho=0)$. The "FG ambiguities" in the expansion determining $G_{\mu \nu}$ will not influence our calculations since they appear one order higher than the expressions we use.

If we use as gravitational action the minimal one, i.e. Einstein term and a negative cosmological constant, then on the solution this action becomes:

$$
S=\frac{1}{\ell^{d}} \int d^{d+1} X \sqrt{\operatorname{det} G_{\mu \nu}} .
$$

As we will discuss in the continuation, for the type A anomalies related to the WZW term we are interested in, (A.2) gives the general expression up to an overall normalization.

The FG gauge is extremely useful since the symmetries playing an essential role for trace anomalies appear as its residual gauge freedom [20] $:^{5}$

a) diffeomorphism transformations which act just on the $x$ variables, inducing therefore the ordinary $d$-dimensional diffeomorphisms on the boundary

b) an additional subgroup of the $d+1$-dimensional diffeomorphisms depending on one function $\sigma(x)$. The transformation (called PBH in the following) leaves the metric in the FG gauge, i.e. $\mathcal{L}_{\xi} G_{\rho \rho}=\mathcal{L}_{\xi} G_{\rho i}=0$, with solution:

$$
\rho^{\prime}=\rho e^{2 \sigma(x)} \simeq \rho(1+2 \sigma(x)), x^{i}=x^{i}-a^{i}(x, \rho)
$$

where

$$
a^{i}(x, \rho)=\frac{\ell^{2}}{2} \int_{0}^{\rho} d \rho^{\prime} g^{i j}\left(x, \rho^{\prime}\right) \partial_{j} \sigma(x) .
$$

Correspondingly, the metric $g_{i j}(x, \rho)$ transforms as:

$$
\delta g_{i j}(x, \rho)=2 \sigma\left(1-\rho \partial_{\rho}\right) g_{i j}(x, \rho)+\nabla_{i} a_{j}(x, \rho)+\nabla_{j} a_{i}(x, \rho) .
$$

The covariant derivatives are with respect to $g_{i j}(x, \rho)$ with $\rho$ fixed.

\footnotetext{
${ }^{5}$ Here our curvature convention are the opposite to those of [20], i.e. $\left[\nabla_{i}, \nabla_{j}\right] V_{k}=R_{i j k}{ }^{l} V_{l}$.
} 
In particular the boundary value of the metric $g_{i j}^{(0)}$ transforms by an ordinary Weyl transformation:

$$
\delta g_{i j}^{(0)}(x)=2 \sigma(x) g_{i j}^{(0)}(x) .
$$

Therefore the PBH transformation lifts the Weyl transformations into an isomorphic subgroup of diffeomorphisms in $d+1$ dimensions. The change between two metrics corresponding to boundary values related by (A.6) can be represented as a $d+1$ dimensional $\mathrm{PBH}$ transformation.

A diffeomorphism invariant action $S$ on a manifold with boundary transforms under a diffeomorphism with parameters $\zeta^{\mu}$ as:

$$
\delta S=\int d^{d+1} X \partial_{\mu}\left(\zeta^{\mu} L\right)=\left.2 \sigma \rho L\right|_{\rho=0}
$$

where $L$ is the gravitational Lagrangian density and we used the PBH diffeomorphism in the $\rho$-direction. Therefore the anomalous Weyl variation of the action is expressed directly through the boundary value and the trace anomalies can be read off directly from (A.7).

To make the discussion more concrete, we give the explicit expressions needed for the calculation of anomalies in $d=4$. The coefficients of the FG-expansion of the metric

$$
g_{i, j}(x, \rho)=\stackrel{(0)}{g}_{i j}(x)+\rho \stackrel{(1)}{g}_{i j}(x)+\rho^{2} \stackrel{(2)}{g}_{i j}(x)+\ldots
$$

are largely fixed by $\mathrm{PBH}$ transformations (A.5):

$$
\begin{aligned}
\stackrel{(1)}{g}_{i j}= & -\frac{\ell^{2}}{d-2}\left(R_{i j}-\frac{1}{2(d-1)} R g_{i j}\right) \\
\stackrel{(2)}{g}_{i j}= & c_{1} \ell^{4} C^{2} g_{i j}+c_{2} \ell^{4}\left(C^{2}\right)_{i j}+\frac{\ell^{4}}{d-4}\left\{\frac{1}{8(d-1)} \nabla_{i} \nabla_{j} R-\frac{1}{4(d-2)} \square R_{i j}\right. \\
& +\frac{1}{8(d-1)(d-2)} \square R g_{i j}-\frac{1}{2(d-2)} R^{k l} R_{i k j l}+\frac{d-4}{2(d-2)^{2}} R_{i}{ }^{k} R_{j k} \\
& \left.+\frac{1}{(d-1)(d-2)^{2}} R R_{i j}+\frac{1}{4(d-2)^{2}} R^{k l} R_{k l} g_{i j}-\frac{3 d}{16(d-1)^{2}(d-2)^{2}} R^{2} g_{i j}\right\} .
\end{aligned}
$$

Here and below $g=\stackrel{(0)}{g}$ and likewise for the covariant derivatives and the curvatures. The coefficients $c_{1}$ and $c_{2}$ parametrize the homogeneous solutions of (A.5) at $\mathcal{O}\left(\rho^{2}\right)$. Inserting this into the expansion

$$
\sqrt{\operatorname{det} g(x, \rho)}=\sqrt{\operatorname{det} g}\left\{1+\frac{1}{2} \rho \operatorname{tr}(\stackrel{(1)}{g})+\rho^{2}\left[\frac{1}{2} \operatorname{tr} \stackrel{(2)}{g}-\frac{1}{4} \operatorname{tr}(\stackrel{(1)}{g} 2)+\frac{1}{8}(\operatorname{tr} \stackrel{(1)}{g})^{2}\right]+\ldots\right\}
$$

one finds

$$
\sqrt{\operatorname{det} g(x, \rho)}=\sqrt{\operatorname{det} g}\left\{1-\frac{\ell^{2}}{4(d-1)} \rho R+\ell^{4} \rho^{2}\left[\gamma C^{2}+\frac{1}{32(D-2)(d-3)} E_{4}\right]+\ldots\right\}
$$

where PBH leaves $\gamma$ arbitrary. ${ }^{6} E_{4}$ is the dimensionally continued four-dimensional Euler density $E_{4}=R_{i j k l} R^{i j k l}-4 R_{i j} R^{i j}+R^{2}$. The contribution to the anomalies can be read off immediately from (A.11).

\footnotetext{
${ }^{6}$ E.g. for the minimal action $\gamma=-\frac{1}{32(d-2)(d-3)}$.
} 
We remark that the type A anomaly is completely fixed by the PBH relation modulo an over all normalization of the action. The same normalization fixes in an unambiguous fashion the $\rho^{0}$ and $\rho^{1}$ terms. This universality of the type A contribution with the accompanying two terms is actually much more general: any modification of the bulk action by higher curvature terms will lead to the same grouping of the three terms, only modifying the overall normalization. This is, in fact, easy to prove.

Consider an arbitrary (local) scalar $\Psi(x, \rho)$ built from the bulk curvature and its covariant derivatives. It will have a FG expansion of the form

$$
\Psi=\Psi^{(0)}+\rho \ell^{2} \Psi^{(1)}+\rho^{2} \ell^{4} \Psi^{(2)}+\ldots
$$

The leading term is always a constant, namely $\Psi$ evaluated on AdS space. Recalling that a term $\rho^{n}$ is accompanied by a curvature scalar of the boundary metric with $2 n$ derivatives, we see that necessarily $\Psi^{(1)} \propto R$ and $\Psi^{(2)}$ must be a linear combination of $\square R, R^{2}, R^{i j} R_{i j}$ and $C^{2}$. Now we use the fact that $\Psi$ was a bulk scalar, i.e. under PBH it transforms as

$$
\begin{aligned}
\delta \Psi & =\xi^{\mu} \partial_{\mu} \Psi=-2 \sigma \rho \partial_{\rho} \Psi+a^{i} \partial_{i} \Psi \\
& =-2 \ell^{2} \rho \sigma \Psi^{(1)}+\rho^{2}\left(-4 \ell^{4} \sigma \Psi^{(2)}+\ell^{2} \stackrel{(1)}{a} \partial_{i} \Psi^{(1)}\right)+\mathcal{O}\left(\rho^{3}\right)
\end{aligned}
$$

where $\partial_{i} \Psi^{(0)}=0$ was used. At $\mathcal{O}(\rho)$ this says that $\delta \Psi^{(1)}=-2 \sigma \Psi^{(1)}$, but there is no such curvature scalar. So we find at $\mathcal{O}\left(\rho^{2}\right)$ that $\delta \Psi^{(2)}=-4 \sigma \Psi^{(2)}$, from where we conclude that $\Psi^{(2)} \propto C^{2}$ and the term proportional to $E_{4}$ is unaffected.

This argument can be generalized to any even dimension.

Therefore the type A anomaly and the related $\sqrt{\hat{g}}$ term are a result of the algebraic structure independent of the bulk action used. For convenience we can use therefore (A.2) with an arbitrary overall normalization. For the type A anomaly in any even dimension (A.2) plays a role analogous to the Chern-Simons action for chiral anomalies. The implications of the "coupling" between the type A anomaly and the $\sqrt{\hat{g}}$ term are discussed in the main part of the paper.

The limiting process $\rho \rightarrow 0$ implicit in (A.7) is not entirely straightforward and we make now more precise some of its features. The actions of the type of (A.2) when evaluated in the FG expansion have pole terms in $d-2 n$ when treated in dimensional regularization. In addition there are a finite number of terms with inverse powers of $\rho$ reflecting the powerlike ultraviolet divergences present formally in the regularization which should be however absent in the effective action of a CFT. If the variation of the action is considered as in (A.7) the pole term in $d-2 n$ is not anymore there and therefore the limit $d \rightarrow 2 n$ can be safely taken, however negative powers of $\rho$ are still present. Therefore before taking the limit in (A.7) we should simply discard these terms. ${ }^{7}$

Alternatively we could use the following equivalent prescription to isolate the trace anomalies: we move the boundary to $\rho=1$ and we use (A.7). There are now in the variation local terms with positive and negative powers of the unique scale $\ell$ as coefficients.

\footnotetext{
${ }^{7}$ Alternatively one can add explicit local boundary terms to cancel them. For a clear discussion consult [23].
} 
The anomaly terms are simply the $\ell$ independent terms in the variation (A.7) (modulo cohomologically trivial terms of course).

Open Access. This article is distributed under the terms of the Creative Commons Attribution License (CC-BY 4.0), which permits any use, distribution and reproduction in any medium, provided the original author(s) and source are credited.

\section{References}

[1] B. Zumino, Y.-S. Wu and A. Zee, Chiral Anomalies, Higher Dimensions and Differential Geometry, Nucl. Phys. B 239 (1984) 477 [inSPIRE].

[2] J. Manes, R. Stora and B. Zumino, Algebraic Study of Chiral Anomalies, Commun. Math. Phys. 102 (1985) 157 [inSPIRE].

[3] J. Wess and B. Zumino, Consequences of anomalous Ward identities, Phys. Lett. B 37 (1971) 95 [INSPIRE].

[4] E. Witten, Global Aspects of Current Algebra, Nucl. Phys. B 223 (1983) 422 [inSPIRE].

[5] M. Henningson and K. Skenderis, The Holographic Weyl anomaly, JHEP 07 (1998) 023 [hep-th/9806087] [INSPIRE].

[6] A. Schwimmer and S. Theisen, Entanglement Entropy, Trace Anomalies and Holography, Nucl. Phys. B 801 (2008) 1 [arXiv:0802.1017] [InSPIRE].

[7] A. Schwimmer and S. Theisen, Spontaneous Breaking of Conformal Invariance and Trace Anomaly Matching, Nucl. Phys. B 847 (2011) 590 [arXiv:1011.0696] [InSPIRE].

[8] Z. Komargodski and A. Schwimmer, On Renormalization Group Flows in Four Dimensions, JHEP 12 (2011) 099 [arXiv: 1107.3987] [INSPIRE].

[9] Z. Komargodski, The Constraints of Conformal Symmetry on RG Flows, JHEP 07 (2012) 069 [arXiv: 1112.4538] [INSPIRE].

[10] I. Low and A.V. Manohar, Spontaneously broken space-time symmetries and Goldstone's theorem, Phys. Rev. Lett. 88 (2002) 101602 [hep-th/0110285] [INSPIRE].

[11] E.A. Ivanov and V.I. Ogievetsky, The Inverse Higgs Phenomenon in Nonlinear Realizations, Teor. Mat. Fiz. 25 (1975) 164 [INSPIRE].

[12] I.N. McArthur, Nonlinear realizations of symmetries and unphysical Goldstone bosons, JHEP 11 (2010) 140 [arXiv: 1009.3696] [INSPIRE].

[13] J.M. Maldacena, The Large- $N$ limit of superconformal field theories and supergravity, Adv. Theor. Math. Phys. 2 (1998) 231 [Int. J. Theor. Phys. 38 (1999) 1113] [hep-th/9711200] [INSPIRE].

[14] R. Kallosh, J. Kumar and A. Rajaraman, Special conformal symmetry of world volume actions, Phys. Rev. D 57 (1998) 6452 [hep-th/9712073] [INSPIRE].

[15] A. Jevicki, Y. Kazama and T. Yoneya, Generalized conformal symmetry in D-brane matrix models, Phys. Rev. D 59 (1999) 066001 [hep-th/9810146] [INSPIRE].

[16] S.M. Kuzenko and I.N. McArthur, Quantum metamorphosis of conformal symmetry in $N=4$ super Yang-Mills theory, Nucl. Phys. B 640 (2002) 78 [hep-th/0203236] [INSPIRE]. 
[17] S. Bellucci, E. Ivanov and S. Krivonos, AdS/CFT equivalence transformation, Phys. Rev. D 66 (2002) 086001 [Erratum ibid. D 67 (2003) 049901] [hep-th/0206126] [INSPIRE].

[18] J.H. Schwarz, Highly Effective Actions, JHEP 01 (2014) 088 [arXiv:1311.0305] [INSPIRE].

[19] H. Elvang, D.Z. Freedman, L.-Y. Hung, M. Kiermaier, R.C. Myers et al., On renormalization group flows and the a-theorem in 6d, JHEP 10 (2012) 011 [arXiv: 1205.3994] [INSPIRE].

[20] C. Imbimbo, A. Schwimmer, S. Theisen and S. Yankielowicz, Diffeomorphisms and holographic anomalies, Class. Quant. Grav. 17 (2000) 1129 [hep-th/9910267] [INSPIRE].

[21] O. Aharony and Z. Komargodski, The Effective Theory of Long Strings, JHEP 05 (2013) 118 [arXiv: 1302.6257] [INSPIRE].

[22] S. Deser and A. Schwimmer, Geometric classification of conformal anomalies in arbitrary dimensions, Phys. Lett. B 309 (1993) 279 [hep-th/9302047] [InSPIRE].

[23] T. Andrade, M. Bañados and F. Rojas, Variational Methods in AdS/CFT, Phys. Rev. D 75 (2007) 065013 [hep-th/0612150] [INSPIRE]. 